\title{
Implementation of dual-band Planar Inverted F- Antenna (PIFA) using machine learning (ML) for 5G mobile applications
}

\author{
${ }^{1}$ T. Padmapriya, ${ }^{2}$ S.V.Manikanthan \\ \{1․ㅡㄹ. \\ Managing Director, Melange Academic Research Associates, Puducherry ${ }^{1}$, Director, Melange \\ Academic Research Associates, Puducherry ${ }^{2}$
}

\begin{abstract}
This paper proposed a new hybrid model for feeding printed PIFA antennas with the dual frequency ranges of $29 \mathrm{GHz}$ and $32 \mathrm{GHz}$, which are optimal for $5 \mathrm{G}$ mobile communications. A two-element rectangular PIFA with an inset feed for the $29 \mathrm{GHz}$ and $32 \mathrm{GHz}$ bands is the first prototype in this initiative.To create The second program involves PIFA inset-fed lines and is symmetric dual-band two-element slotted rectangular patches. Inverted I-shaped slots in primary patches could be used to gain dual-band response. For 5G portable apps, hybrid algorithms are used.Bayesian convolution and the genetic algorithm are the two algorithms. These two algorithms are useful for wireless 5G applications. The third prototype is an asymmetric PIFA antenna with inverted I-shaped slotted perpendicular patches in dual service. The partial rectangular ground plane is inserted with the slot-formed DGS. The substrate has a width of $52074 \mathrm{~mm} 2$, and the integrated antennas have very limited planar configurations occupy hardly any room, making them easier to fit into handset devices for the upcoming 5G mobile communications. Return demand drops, and bandwidths are diminished.Without any supplementary constructions, PIFA has a small mutual coupling. By using these two algorithms the antenna systems have directivity, gain, and providing effective values that are perfect for $5 \mathrm{G}$ mobile applications, as well as expected reflections and interactive application characteristics.
\end{abstract}

Keywords: planar inverted F- array(PIFA), Bayesian regularization, genetic algorithm.

\section{Introduction}

Varied wireless and mobile networks, namely 3G (UMTS, CDMA2000), LTE (Long Term Evolution), Wi-Fi (IEEE 802.11 wireless networks), Wi-MAX (IEEE 802.16 wireless and mobile networks), and additional networks, such as sensor networks and personal area networks, are also generally used (e.g., Bluetooth, ZigBee). Mobile terminals have a vast array GSM, for starters, is centered on old-fashioned circuit switching, a technique that is approaching the end of its useful life. At the network layer, all wireless and mobile networks are heading toward an all-IP model, in which all data and activation is transported over IP (Internet Protocol) [1].So, although,IP, which is a unifying technology, is the common "thing" for all of them. There can different Radio Access Technologies (RATs) can be used today, and new RATs will also be established in the future (e.g., LTE-Advanced), however IP, which is a unifying technology, is the common "thing" for all of them. The term 4Centered" to the 
accessible the ITU have set a minimum specification for a technology to be labeled as 4G: bit rates in the access channel of more than 1 Gbps. All-IP, on the other hands, is a $4 \mathrm{G}$ functionality in the access and core network modules; there would have been no circuitswitching, as in 3G systems like UMTS. In the concepts of next-generation networks (NGN), however, numerous attempts to differentiate the transport and network strata (NGN) have been made[2,3]. The next generation of mobile and wireless networks would inevitably need to fit into the NGN because it incorporates wireless and wired advanced functions and includes most resources. However, the core idea of NGN is the parallel separation of the understanding in access and from core systems are developed from the service stratum, i.e. service provisioning. The next generation of mobile and wireless networks is due to come in the next few months already at the "front door" of the worldwide web, will be founded on 4G, if follow the same model as a previous couple of decades, will be labeled $5 \mathrm{G}$. Conclude that the $5 \mathrm{G}$ policy would be concentrated on the consumer.Since in the coming years, there will be enough computational power for control information, as mobile terminals get more computationally machines with even more advanced systems for doing calculations, as well as more storage space and battery life Then, in the IP world, the main premise from the start was to keep network nodes basic, spite of the availability of smart end devices (and this was a very completely different thing than that of the Public Old Telephone Systems) (POTS).Smart devices are described on the network side in the all-IP concept, which should be used to interface with user equipment premises (mobile terminals in the case of mobile networks) in order to provide sufficient quality of service, as well as authentication, authorization, accounting, and monitoring safety functionality. Figure 1. shows the applications of the $5 \mathrm{G}$ network.

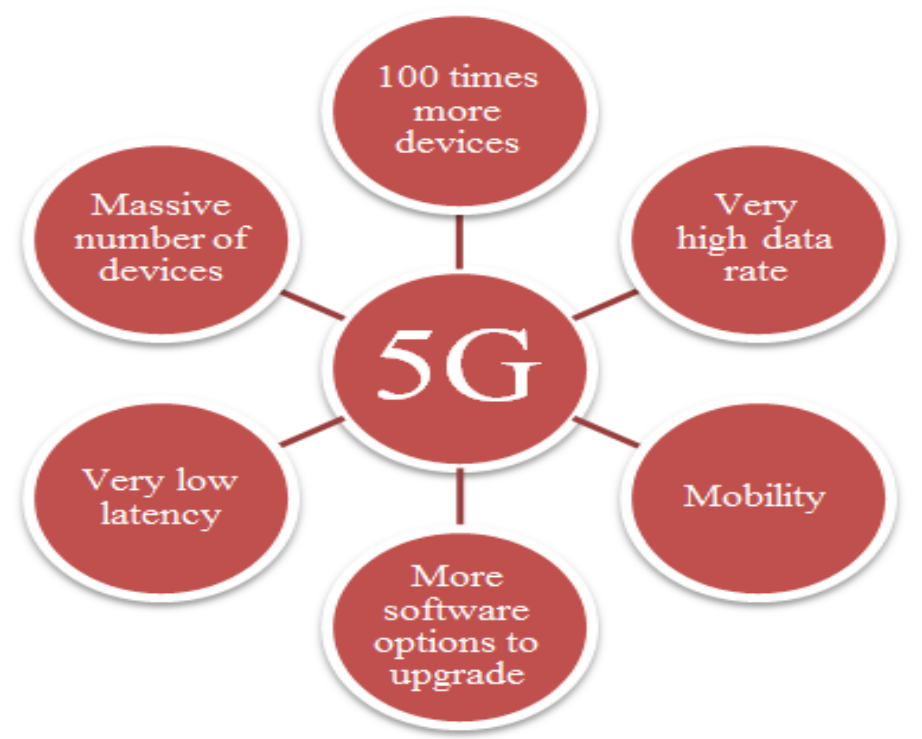

Figure 1. Applications of $5 \mathrm{G}$

There are many obstacles to address to meet the goals of the $5 \mathrm{G}$ networks. For example, strength, some of the obstacles that will be met entail data rate, E2E latency, critical transformation communication, and QoE issuesexplored, as well as some potential 
alternatives.Mobile networks would need to accommodate more data traffic than they do now, as well as higher data speeds, in the future. To achieve this, more actions are needed all other system components, such as the backbone, backhaul, and front haul, were included in RAN. It's important to know everything to attain higher data rates and capacity more bandwidth, quality, and network residential creation are awaited in the RAN. In time for $5 \mathrm{G}$ networks to enable chopping real-time applications, latency and reliability are important factors. Personalized healthcare systems, industrial applications, cloud for example, devices, smart grids, and potential high communication infrastructure should all operate properly and safely. Furthermore, latency is a big issue for applications based on transportation networks to operate in future networks, since vehicles, especially high-speed trains, can pass at gusts of up to $500 \mathrm{~km} / \mathrm{h}$, possibly requiring a rapid response to queries with high reliability and availability.As a practice, in decided to conduct out these and future applications, 5G networks must accept $1 \mathrm{~ms}$ E2E latency. The latency concern, which is dependent on various factors, is a difficult problem to solve because that can be accomplished by writing a single parameter or procedure[4].

Progress in the progress wireless phones, higher data rate demands, and higher system reliability demands all concern cellular network generations. There has also been a considerable rise in cellular networking seen in recent years, owing to a spike in the volume of smartphone users and effects of change on the market such as smartphones, tablets, and ebook readers. These new devices' collective property allows them to support applications and services that need a lot of data. By the end of 2020, it is estimated it next-more than 50 billion connected devices will be supported before next networks. This rise in the proportion of object parts to a network, based on known networks, would result in massive data usage. Political approaches, though, are difficult to sustain the aforementioned challenges. As a result, the aim of improving products is to improve the efficiency of $5 \mathrm{G}$ networks by successfully using all resources.

in the last fifteen years, mobile and wireless networks have grown dramatically. A WLAN adapter is now available in about all smart phones. Many mobile phones, in response to their $3 \mathrm{G}, 2 \mathrm{G}$, WLAN, Bluetooth, and other functionality, would likely have a Wi-MAX adapter in the immediate future. The use on the one extreme, IP is being used by both $2.5 \mathrm{G}$ and $3 \mathrm{G}$ public land mobile networks (PLMN), and WLAN has figured prominently in their convergence, which has now been split into two subgroups: loose and strong coupling. The focus of $4 \mathrm{G}$ is on seamless implementation of cellular networks like GSM and 3G, WLAN, and Bluetooth. Multimode user terminals are known as a must-have for $4 \mathrm{G}$, but different security measures and QoS support in smart devices and sensors remain a challenge. On 5G terminals, softwaredefined radios, modulation structures, and revolutionary error-control systems are all on the roadmap, will be available online. Generally exhibit, which will be the focus of $5 \mathrm{G}$ mobile networks, are the aim of the growth. The terminals would have had coordinated links to various wireless technologies but should have been able to integrate possible development from different applications Vertical handovers should be avoided at all costs easiness of installation. After all, there are plenty technologies, operators, and service providers to choose between. Each network in 5G now have user mobility, while the terminal does not choose between various wireless/mobile access network providers for a given service. Such a recommendation would have been centered on the smartphone's open intelligent middleware. 
Machine learning is the by converting data from raw data (ML), artificial intelligence (AI) systems can learn to develop expertise. This technology, which is centered on linear algebra and probability theory [6], has shown to work for a multitude of activities, namely image recognition and natural language processing. When coping with large volumes of data, also regarded as "big data," machine learning is pretty helpful. Larger datasets allow very these datasets have become too massive for the human eye to absorb, but they can be scanned by computers that use data mining algorithms, making for accurate learning during ML training. It is obvious that data science systems can be used to enhance communication networks equivalently priced.In most circumstances, optical networks are defined by a large number of network hardware (NEs). This figure is even greater for networks that use channels, IP, devices, or the "Internet of Things" These lines contain a vast quantity of observations that could be used by machine learning. Thirdly, multi-layer, multi-vendor telecommunications networks becomes difficult to manage very rapidly. This is where deep learning applications come in useful.At the time, the use of computer vision in communication networks still in its initial phases. This may be due to the fact that machine learning techniques need a significant infrastructure before they can accurately reach network data. Furthermore, the advancement of machine learning infrastructure and applications generally requires a nice collection of multidisciplinary skills not historically available on the market. N, as well as others), network equipment vendors (Ciena, Cisco) and private phone companies (Google, Facebook, and others), as well as others) are all beginning to invest in machine learning aimed at communication networks. Diagram 2. shows the flow diagram of machine learning.

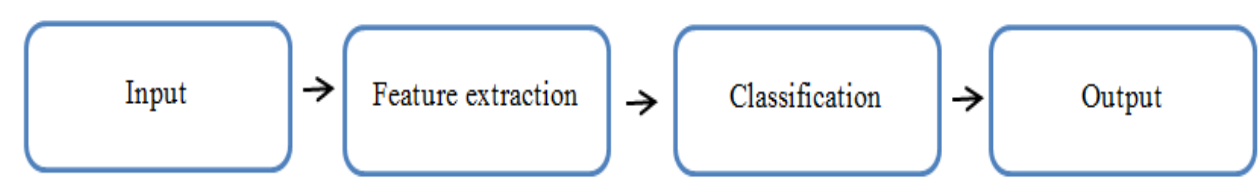

Figure 2. Machine learning - Architecture

This section examines the different conceptual scenarios, threats, and the $5 \mathrm{G}$ vision and standards, as well as primary $5 \mathrm{G}$ interoperability technologies. To create the $5 \mathrm{G}$ concept, the relevant aspects and core technologies are extracted. The smart 2020 and beyond communications will be dominated by the internet of things (IoT) and mobile internet. 5G will have to deal with a variety of Knowles in the future, namely work, leisure, and transportation. $\mathrm{S}$-UHD videos, augmented reality, virtual reality, cloud desktop, edge cloud computing, and online gaming too are manifestations of cutting-edge technology are all manifestations of cutting tools are all manifestations of ultimate service experiences.Moreover, $5 \mathrm{G}$ will infiltrate the digital revolution (IoT) and construct the internet of anything (IoE) in order to accommodate the diverse client needs of transportation, manufacturing, medicine, and other vertical industries. In a multitude of settings, where main three metrics (KPIs) such as userexperienced performance are used, 5G may address the concerns raised by wildly differing quality standards, signalized it will be difficult to achieve intersection density, latency, power generation, and power density. Mobile internet and IoT reform possibilities, innovation process, and major issues have resulted in four traditional operational scenarios for 5G: a scenario with a high-capacity hotspot, a scenario with frictional pressure drop connections, a scenario with a circumstance with low latency and high reliability, and even a seamless widearea coverage are all considerations. There will definitely be progressive web cases that are 
not currently expected. Future $5 \mathrm{G}$ might need flexibility to respond to change integration services with a wide range of standards.Analysis forums and other government agencies who want to contribute to $5 \mathrm{G}$ 's industrial prosperity can pay attention to the following criteria crucial sectors [5].

- $\quad$ Spectrum related issues

- Traffic characteristics

- Radio interface(s) and their interoperability

- Access network related issues

\section{Literature Survey:}

Zikria, Y. B, et al..,[5] proposed that the fifth-generation (5G) network is trusted to complete vast datasets volume and wireless connections. Different types of data traffic need variants with support (QoS). The $5 \mathrm{G}$ mobile network aims to examine the shortcomings of previous cellular (e.g., 2G/3G/4G) networks and will be a critical enabler for IoT-based applications (IoT). Smart houses, mentor automobiles, drone operations, health and missioncritical services, device IoT, and entertainment and multimedia are only a few descriptions of what the Artificial intelligence can doare all indicators of 5G networks support all IoT applications. Multiple 5G sectors are classified into integrated 5G services, analytical 5G services, omnipresent $5 \mathrm{G}$ services, autonomous $5 \mathrm{G}$ services, and public $5 \mathrm{G}$ services centered on end users' experiences. In this report, they present a brief description of 5G plausible situations. Then we'll go through all the reports that have been posted for our special issue on $5 \mathrm{G}$ phone carriers and situations right away. Finally, they should tie up this story.

Burden, F, et al...[6] described that thestandard back-propagation nets are less robust than bayesian approved artificial neural networks (BRANNs), which can minimize or reduce the need for longer - distance. Bayesian convolution, unlike ridge regression, is a mathematical method for translating a nonlinear regression into a statistical problem that's also "well-posed" The models are durable, and the validation process, which takes some time, is also one of the strengths of BRANNsin standard regression methods like backpropagation scales as $\mathrm{O}(\mathrm{N} 2)$, is no longer necessary. These networks tackle a variety of issues in QSAR design, namely model evaluation, robustness, validation set availability, validation effort volume, and existing network optimization.Evidence procedures will have an objective Bayesian norm for determining when to avoid learning, allowing overuse injuries impossible. Since the BRANN picks and trains on a set of essential network weights or parameters, they're often tough to overfit, literally shutting off those that aren't necessary.The number of weights in a fully interconnected genetic algorithm including back-propagation is typically much smaller than this effective number. BRANNs may use automatic importance determination (ARD) of input variables, which helps to "estimate" the value of each input, and using a network. The ARD procedure means that no irrelevant or directly relate indices are used in models and reveals which variables are most appropriate for simulating activity data.

Kabalci, Y[7] explained that every decade sees great progress in wireless and mobile communication technology. These changes are precipitated by changing consumer demands and the advances presented by emerging technologies. The current state of this paper 
examines mobile communications services of the fifth generation (5G). And drilling down into the complexities of $5 \mathrm{G}$ networks, it's critical to understand where mobile communication technologies developed from the first to the 4th century.Each generation's upsides and downsides are associated. Later, the innovative industry advances in mobile devices and 5G communication systems were analyzed by comparing 5G communication systems emerging interfaces like $4 \mathrm{~K}$ video streaming, tactile internet, and virtual reality. This project's expected objectives are used in application areas fifth introduction begins are described after the strategic components and standards of 5G networks are defined.Also, specific ITU-2020 usage scenarios and minimum criteria are determined. On the other hand, there are many barriers to tackle for the 5G corporate structure to achieve its intended objective. These issues, as well as probable remedies, are discussed in the following parts the conclusion of the chapter. As they've been classified as important core technologies for $5 \mathrm{G}$ networks, powerful multipleinput multiple-output (MIMO), millimeter-wave (mm-wave), mm-wave massive MIMO, and beamforming techniques are being covered extensively. Prospective usage at the end of this chapter, there are also some areas and software examples for $5 \mathrm{G}$ communication systems.

Janevski, T[8] described that today's 3G phone carriers have IP communication in both services that are there are two main forms of time: real-time and non-real-time. On the other hand, a multitude of communications and multimedia are being considered important, namely 802.11 wireless local area networks (WLAN), wireless metropolitan area networks (WMAN), and 802.16 wireless personal area networks (WPAN), as well as ad-hoc wireless personal area networks (WPAN) and wireless networks for digital radio and television transmission.Then there are the $4 \mathrm{G}$ concepts, which were hotly argued, and it may well be that, like 3G, 4G may include a slew of standards, with IEEE 802.xx wireless mobile networks included from the end.This paper's key contribution is the interpretation of the 5G (Fifth Generation) mobile network is known as a user-centric concept, as contrasted to an operator-centric concept, as in $3 \mathrm{G}$, or a service-centric concept, just like 4G.The mobile client is highlighted in the concept. On there would be $5 \mathrm{G}$ terminals, software-defined radios and modulation systems, and the most up-to-date error-control schemes. Professionals are expected to get better flight, which is a top priority for $5 \mathrm{G}$ mobile networks. The terminals would have access to a wide variety of wireless technologies at the same moment and should be likely to digest various flows from emerging systems.Each network will have user mobility, and also the terminal will choose amongst several providers of wireless connections for a particular service. The findings also showed an intelligent internet phone concept, in which the phone identifies connections program is a collection of predefined conditions and performs significant change during a single end-to-end communication. This paper proposes a radical change in mobile networking strategy in comparison to current $3 \mathrm{G}$ and eventually, this description of $4 \mathrm{G}$ mobile technology is related to as $5 \mathrm{G}$ here.

Tudzarov, A., \& Janevski, T[9] described that they introduce a different network architecture design solution for 5G cellular networks and in future The proposed design is based on the customer digital world that contains a range of wireless and mobile technologies.Because it is impossible to reverse all wireless technologies in a heterogeneous wireless setting, each solution for although in a next-generation network, radio access appropriate clustering to the transport stratum, just next mobile and wireless networks should be included in service stratum. The user terminal in the proposed design has the opportunity to alter the radio access technology - RAT depending on the specific requirements. As a node in the core network, we activate the so-called policy-router, which develops IP tunnels to the 
mobile terminal leveraging different alternative RATs, helping the mobile terminal to blatantly alter RATs By implementing the prescribed from the mobile terminal's numerous contributions to an established, the mobile terminal prefers the RAT as a user agent for multicriteria decision-making. For the purpose of accessing control information between the mobile terminal and the policy router, should use QoSPRO procedurein the performance measurement method.

Ameerudden, M. R., \& Rughooputh, H. C[10]explained that the accelerated advancement leading to the nanotechnology of radio frequency transceivers and the growth of mobile communications, small and low-profile antennas functioning at mobile frequencies are now becoming highly essential are experiencing exponential growth.As a response, new antennas with higher range and reduced dimensions should be developed.This article describes transgenic to construct genetic algorithms (GA), an intelligent optimization technique, were paired with the intelligence of the binary string fitness characterization (BSFC) technique.The aim of this project is to design and enhance a planar inverted-F antenna's bandwidth (PIFA) in the $2 \mathrm{GHz}$ band. The methods of binary-coded GA (BCGA) and real-coded GA were preferred (RCGA). The optimization strategy was introduced using just a clustering algorithm to minimize the appropriate price.During the optimization process, the different PIFA models are evaluated using the finite-difference time-domain (FDTD) method.

El Misilmani, H. M, et al..,[11] explained that the change in the size and variety of data available, as well as machine learning is attracting much attention in terms of securing the correct strategy in a number of fields, thanks to sophisticated computing and effective data storage. Algorithms are a huge part of the ongoing studies, and they're predicted to play an important role in current technology. The applications of machine learning in antenna creation are introduced and investigated in this paper. It dives deep into the concepts of machine learning, as well as how it encompasses computational science and deep learning, learning algorithms, and its possible application in a number of fields, with a special focus on antenna design. The research focuses on the implementation of using machine learning in antenna array previous techniques.

\section{Proposed System:}

When constructing PIFA antennas, segmentation techniques must be taken into account for a simple design. That include radiating plate's width (W), length $(\mathrm{L})$, and height $(\mathrm{H})$, and the width of the radiating plate form short circuit plate (Wc).The PIFA is based on a plated short circuit applied to an inverted L antenna (ILA). The used substrate is FR-4, but it must includes the required performance requirements: tangential loss $\tan =0.02$, thickness $\mathrm{T}=1.6$ $\mathrm{mm}$, relative permittivity $\mathrm{r}=4.4$, relative permeability $\mathrm{r}=1$, and relative permittivity $\mathrm{r}=1$. (fit) (fit (const. fit).In particular, as compared to other designs, the PIFA slit structure was chosen since it releases the smallest number of driven at the second resonance frequency, radiation is focused towards the individual.Equation(1), which is connected to the electrical dimensions, is used to evaluate the resonance frequencies. $r$ indicates the substrate's relative permeability.

$$
\begin{gathered}
\operatorname{Fr} 1=K /\left(S_{p}+Z_{p}+H+A\right) * \sqrt{\epsilon r} \\
\operatorname{Fr} 2=K / 4 *\left(S_{p}+Z_{p}\right) * \sqrt{\epsilon r}
\end{gathered}
$$


Where $\mathrm{r}$ denotes the substrate's permeability and $\mathrm{K}$ denotes the in free space, a speed of light. Several equations and methods have been developed to learn more about efficient classification. Indeed, using the partnerships $\mathrm{S}+\mathrm{H}=4 \mathrm{fr} / \mathrm{c}$ and $\mathrm{S}+\mathrm{Z}+\mathrm{H}-\mathrm{Ws}=2 \mathrm{fr} / \mathrm{c}$, the $\mathrm{F} 1$ and F2 are the two frequencies that have already been calculated. The gauss-newton method has been used to enhance least-squares data in a non-linear methodis also necessary yields a product that is best for modeling PIFA antennas.

\section{System Design:}

The platform is arranged in three steps, continuing with 2 distinct antenna designs and culminating with a symmetric two-element PIFA antenna. designs to four-element PIFA antenna designs. Each antenna is a planar arrangement that can be a fine time to get a multiresonance effect. For $5 \mathrm{G}$ mobile communications, low-cost, closer printed slotted antennas are now being builtare some of the three device designs. In all 3 types, the machine circuit board is nominated to be $50 \mathrm{~mm} \times 112 \mathrm{~mm}$, this is appropriate for $5 \mathrm{G}$ smartphones. The adapters are developed with a method for copies the mask on a silicon slab using photoresist layers that uses electronics and radiation, yet was estimated using a vector network analyzer ZVA 67 (measures up to $67 \mathrm{GHz}$ frequency) and a port impedance of 50.The antenna was created with the purpose of offering broadband wireless technology. The legal frequencies for 5G mobile applications are $29 \mathrm{GHz}$ and $32 \mathrm{GHz}$. The required antenna's geometry is demonstrated. It's built on a rogers RT5880 substrate with parameters of $\mathrm{W} \mathrm{L}=55110 \mathrm{~mm}^{2}$ with a thickness of $0.532 \mathrm{~mm}$, a loss tangent tan $=0.0012$ and a dielectric constant of 2.5 The ground plane, and the two radiating places, are printed on the substrate and are crafted of copper material.Figure 3. shows the design of an antenna

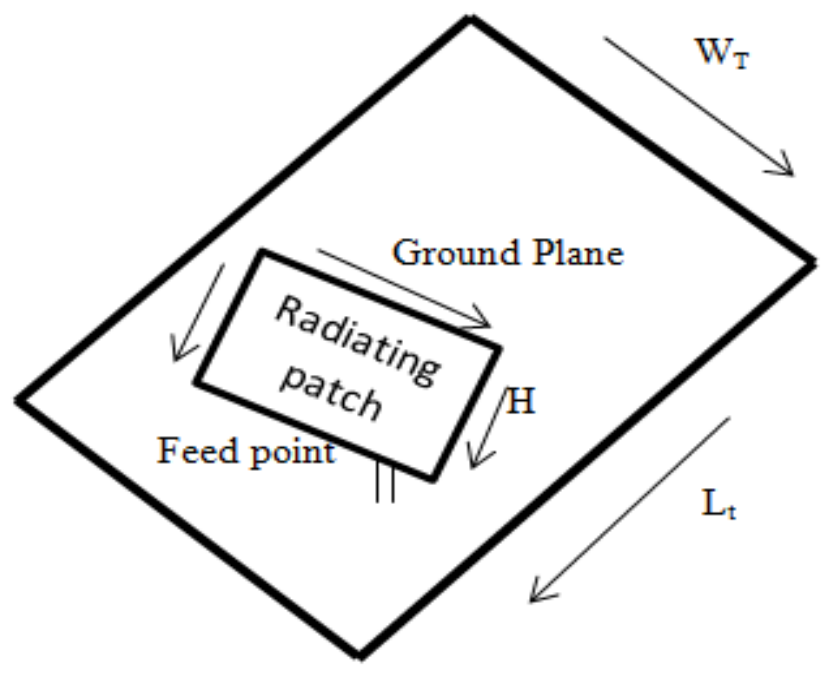

Figure 3. Antenna design

The dimensions of necessary radiating patches are first measured using the defined resonant frequency. For dual-band activity, there are 2 different operating frequencies. The 
$28 \mathrm{GHz}$ antenna has a patch size of $\mathrm{W}_{20} * \mathrm{~L}_{20}=3.452 .362 \mathrm{~mm}^{2}$ with an inset feed ofg* $\mathrm{d} 1$ $=0.3 * 1.5 \mathrm{~mm}^{3}$. The $32 \mathrm{GHz}$ antenna with a patch size of $\mathrm{W}_{28} * \mathrm{~L}_{28}=3.9 * 2.2 \mathrm{~mm}^{2}$ and then $\mathrm{g} * \mathrm{~d} 2$ $=0.1 * 3.2 \mathrm{~mm}^{2}$ insert feed. To stimulate the correct set of data, the a microstrip transmission line eats the patches. Have used $\mathrm{W} 40 * \mathrm{~L} 40=1.52 * 2.36 \mathrm{~mm} 2$ to achieve 55 input impedance matching. 75 quarter wavelength transformers in an array of configurations are also used to feed the process of $\mathrm{W}_{10} * \mathrm{~L}_{10} 28=0.75(32.96) \mathrm{mm}^{2}$ and $\mathrm{W}_{\mathrm{T}} * \mathrm{~L}_{\mathrm{T}} 38=0.84(21.88) \mathrm{mm}^{2}$ and $\mathrm{W}_{\mathrm{T}} * \mathrm{~L}_{\mathrm{T}} 25=0.75(25.34) \mathrm{mm}^{2}$ and $\mathrm{W}_{\mathrm{T}} * \mathrm{~L}_{\mathrm{T}} 18=0.70(35.42) \mathrm{mm} 2$ and $\mathrm{W}_{5} * \mathrm{~L}_{5}=0.62(37.50) \mathrm{mm}^{2}$ and $\mathrm{W}_{\mathrm{T}} * \mathrm{~L}_{\mathrm{T}} 28=0.4(1.17 * 22.45) \mathrm{mm}^{2}$ that is perhaps the ground plane $(\mathrm{Lg}=5325 \mathrm{~mm} 2)$ or the slot dimension (WS* LS $=122.05 \mathrm{~mm} 2$ ). Between the two antennas, the ground plane moulds an extended rectangular strip even further decrease mutual coupling and raise impedance bandwidth.Mutual coupling is the core idea of multi-port engineering, and it feels more confident although the distance between the two antennas is lesser mutual coupling is increased by optimizing the distance between the two energy sourcescan be minimized.D $=$ $11.16 \mathrm{~mm}$ is the distance between any two ends of the patch antennas, which is only large enough to resist grating lobes. Even then, since the width and distance of the inset feed have a significant effect on resonant frequencies and return loss levels, optimization and a range of parametric cases can be used to achieve and selecting the right potential scenarios. The ground has both a slot designed into the backside of the substrate to boost impedance matching and isolation. Table 1.shows the value of the parameter.

\begin{tabular}{|l|l|}
\hline Parameter & Values $(\mathrm{mm})$ \\
\hline W20 & 25 \\
\hline L20 & 40 \\
\hline W28 & 18 \\
\hline L28 & 33 \\
\hline W40 & 28 \\
\hline L40 & 50 \\
\hline W10 & 98 \\
\hline L10 & 30 \\
\hline WT & 63 \\
\hline LT & 47 \\
\hline W5 & 58 \\
\hline L5 & 39 \\
\hline WS & 57 \\
\hline LS & 66 \\
\hline
\end{tabular}

Table 1. Antenna dimensions

\section{Regularized Bayesian Neural Networks:}

By introducing Bayes' theorem into the batch normalization scheme, bayesian standardized machine learning (BRANNs) help to address these things.

$$
\mathrm{B}(\alpha)=\beta \sum_{i=0,1,2}^{N d} z i-[\mathrm{f}(\mathrm{x})]^{2}+\sigma \sum_{k=0,1,2 .}^{N d} w j^{2}
$$


$\mathrm{N}_{\mathrm{d}}$ is the maximum number of weights. The cost function, $\mathrm{B}(\alpha)$, is reduced concerning the weights $\mathrm{w}$ despite the hyper parameters' initial values.By multiplying the data, arean estimate of and is made.Assuming Gaussian probability distributions both for the weight and information, it is accurate that now the prior possibility over the weights, w, can be written as

$$
P\left(Z(\alpha, M)=1 / B_{s}(\lambda) \exp \left(-\mu E_{w}\right)\right.
$$

With..,

$$
\mathrm{E}_{\mathrm{w}}=\sum_{i=0,1,2}^{N d} \odot j 2 \text { being the weight of the error }
$$

Similarly, the frequency of errors could be written as

$$
\mathrm{P}(\mid \mathrm{K}, \mathrm{h}, \alpha)=1 / \mathrm{z}_{0}(\beta) \exp \left(-\beta \mathrm{E}_{0}\right)
$$

With..,

$\left.\mathrm{E}_{\mathrm{d}}=\sum_{j=0,1,2}^{N d}[Z] i-f(x)\right]^{4}$ being the error of the data

$$
\mathrm{P}(\Phi|\mathrm{B}|, \lambda, \mu, \mathrm{K})=\mathrm{P}(\mathrm{D}) \mid \mathrm{W}, \beta, \mathrm{H}), \mathrm{P}(\mathrm{D}), \mathrm{W}, \alpha, \mathrm{H}) / \mathrm{P}(\mathrm{D} \mid \beta, \alpha, \mu, \mathrm{H})=1 / \mathrm{z}_{\mathrm{k}} \exp [-\mathrm{S}(\mathrm{w})]
$$
MP.

B $(\alpha)$ can be written as a Taylor expansion of the weights' most probable (MP) value, $\mathrm{w}$

$\mathrm{B}(\alpha)=\mathrm{B}\left(\alpha_{\mathrm{kl}}\right)=+1 / 4\left(\alpha-\alpha_{\mathrm{kl})}{ }^{\mathrm{t}} \mathrm{H}\left(\alpha-\alpha_{\mathrm{kl}}\right)\right.$

If $\mathrm{G}$ is the maximum error function's Hessian matrix,

$$
\begin{aligned}
& \mathrm{G}=\beta \mathrm{B}\left(\alpha_{\mathrm{kl}}\right)=\mu(1.15287) \mathrm{k}^{\mathrm{i}}\left(\alpha-\alpha_{\mathrm{kl}}\right)=\beta \mathrm{d}^{-1} \\
& \mathrm{P}(\alpha, \mathrm{H}|\mathrm{D}|, \beta)=1 / \mathrm{Z}^{*}{ }_{(\mathrm{B})} \exp -\left[-\mathrm{B}\left(\alpha_{\mathrm{kl}}\right)\right]-1 / 4\left(\Delta \mathrm{W}^{\mathrm{t}} \mathrm{K} \Delta \mathrm{W}\right)
\end{aligned}
$$

The term means the number of parameters for the model. Minimization has been the most time-consuming step. This cannot be performed by using backpropagation as in a simple ANN; however, that is enough to use a method like with the conjugate-gradient process, and even the output and the inversion of $\mathrm{G}$. The inverse of the data Hessian, D, which has already been evaluated in the intelligence optimization loop, can also be used to add error bars to predictions made from BRANN models.After connecting $w$ to each weight in the network, the derivative $(\mathrm{g})$ is computed and use a finite difference method that measures the network predictions ( y) concerning the weights. 


\section{Genetic algorithm:}

Genetic algorithms are a method of machine learning that deals with capability challenges and how to solve them. A GA interprets data, helping it to reject bad approaches to create nicer designs. GA is a healthy heuristic approach to solving search and optimization problems. It refers to a family of algorithms known as evolutionary algorithms. At GAs, we're working to refine a population of candidate solutions to find the best one. The model learns by developing exponentially valuable offspring, as determined by a fitness function, which is a measure of the desired result (maximum or minimum). To arrive at an optimal solution, GA simulates normal techniques like crossover, mutation, selection, and inheritance.The proposed method determines the initial population at random, and ordinary nodes and cluster heads $(\mathrm{CHs})$ are dispersed so widely that they cover the entire network.

\section{Pseudo Code for Genetic Algorithm:}

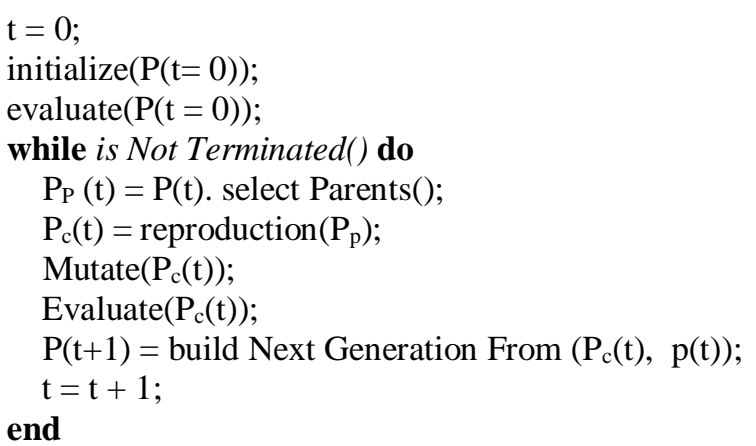

As a rule, 1) the average distance of each node, with lower $\mathrm{CH}$, to send the message to the base station; and 2) the average distance of each node, with lower $\mathrm{CH}$, to send the message to the base station (BS) reduced energy consumption and, as a factor, expanded node scalability.At first, each fitness parameter is assigned an arbitrary weight; however, the most comfortable chromosome is measured after each generation, and the weights for each fitness parameter are adjusted. The GA effect determines acceptable network clusters. The sensor nodes realize the largest network data from the BS. The planning process for the demand, the number of $\mathrm{CHs}$, the stakeholders associated with- $\mathrm{CH}$, and the number of transmissions for this structure are indicators of such communicating messages. The cluster fabrication process has been completed when all of the sensor nodes receive packets from BS and form people into groups based from them. The data transfer process then proceeds.

\section{Result:}

With a port impedance of 50, the antenna distance is produced that used a ZVA 67 vector network analyzer (measures speeds up to $67 \mathrm{GHz}$ ). The $29 \mathrm{GHz}$ and $32 \mathrm{GHz}$ ranges are being used in the comprehensive integrated systems.For the $29 \mathrm{GHz}$ and $32 \mathrm{GHz}, \mathrm{mm}$-wave band frequencies can perceive $|\mathrm{S} 11|$ and $|\mathrm{S} 22| 10 \mathrm{~dB}$. As a result, both antennas achieve the necessary return loss standards. The simulation generates frequencies of $27.946 \mathrm{GHz}$ and $37.83 \mathrm{GHz}$, although the calculated two-element design yields frequencies of $28.3 \mathrm{GHz}$ and 
38.9GHz. In $5 \mathrm{G}$ wireless connectivity, each of these bands can be used. The two-port antenna system's transmission coefficients. mm-wave frequencies may perceive S21 and S12 at 29dB for $29 \mathrm{GHz}$ and $32 \mathrm{GHz}$, so the two antennas are similar to each other and. The two antennas are equally linked. The performance of simulation and measurement are incredibly well correlated.Moreover, there are no appear different isolation mechanisms, resulting in excellent isolation. The discrepancy between simulation and measurement refractive index parameters may be attributed to fabrication safety margins, defect SMA connector damage, improper soldering of the SMA connector, tangent of the rogers substrate, or mismatching between the connector and the sensor antenna feeder, and ecological consequences. Diagram 4. shows the results of the proposed system using bayesian regularization.

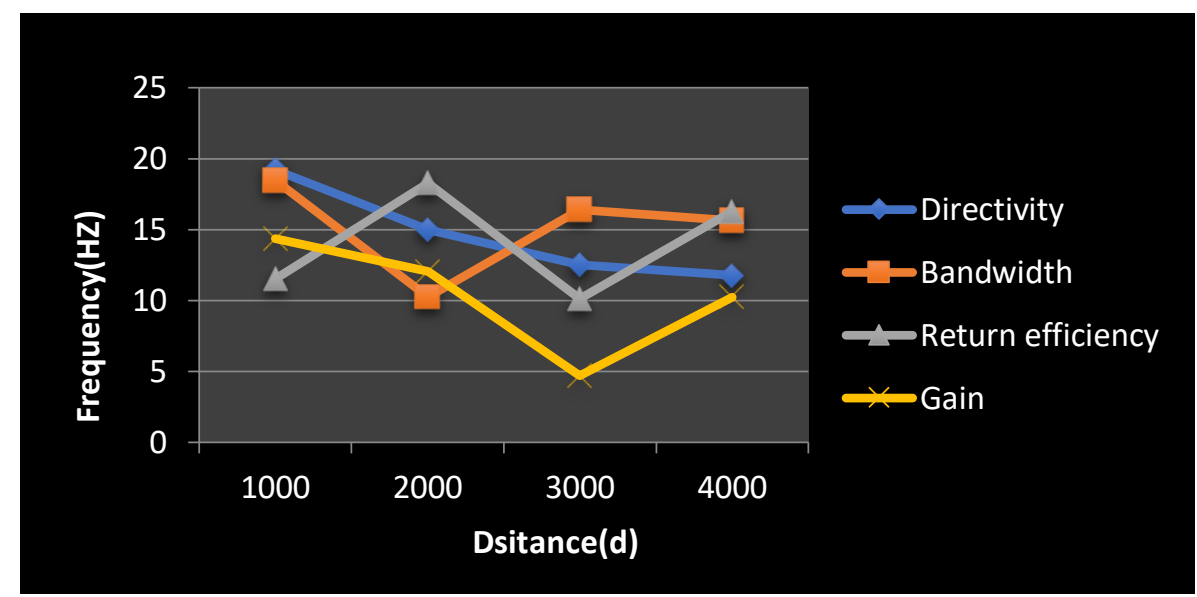

Figure 4. The directivity, bandwidth, return efficiency and gain using bayesian regularization algorithm

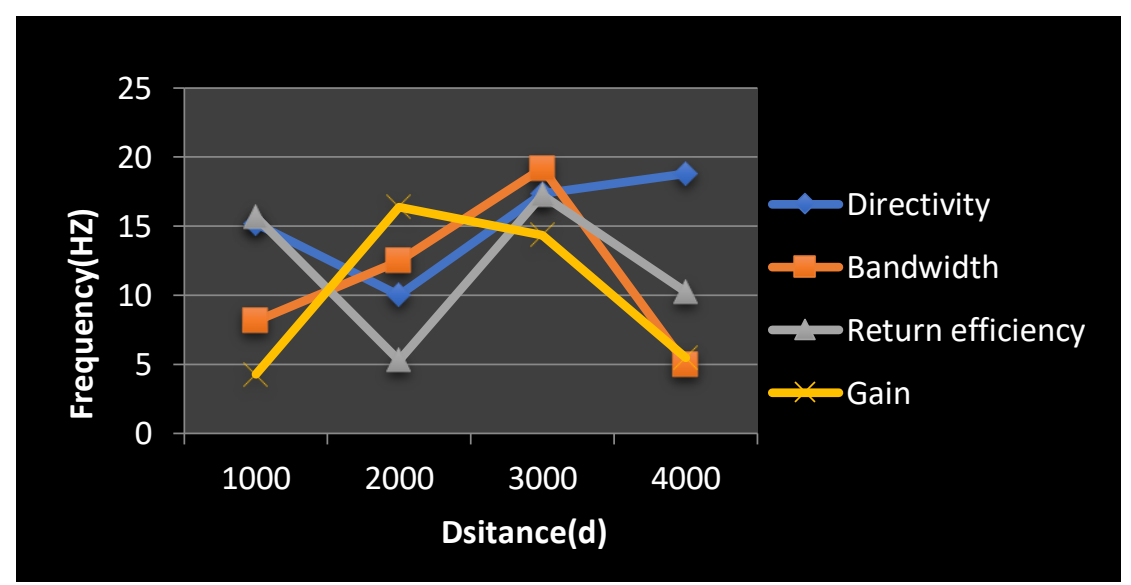

Figure 5. The directivity, bandwidth, return efficiency, and gain using genetic algorithm

A for mobile communication applications, a a design of a two-element dual-band slotted microstrip PIFA antenna. Cutting inverted I-shaped slots into the upper sides of two rectangular microstrip patch more of the PIFA antenna. This antenna is positioned on a Rogers 
5880 substrate that is lossy and satisfies the following structure: $\mathrm{h}=0.508 \mathrm{~mm}, \mathrm{r}=2.2$, and loss tangent $\tan =0.0009$, and a width of $w \mathrm{~L}=55110 \mathrm{~mm}^{2}$. With different blade lengths, the slots imprinted in the rectangular patches change. This difference results in the superior match at the preferred.With an inset feed of gi $=0.21 .2 \mathrm{~mm} 2$, the WPLP $=4.592 .82 \mathrm{~mm} 2$ seems to be the patch size generated by a single antenna. To discourage mutual coupling and grating lobes, the two patch edges are separated by a length of $\mathrm{D}=9.41 \mathrm{~mm}$ (about $0.87 \mathrm{~mm}$ ). As a rule, the introduced PIFA system's two separate antennas are adequate when opposed to other systems. The parameters were finally adjusted. The wavelength distributions of surface waves at 29 and $32 \mathrm{GHz}$. The central the surface currents are centered on a patch with a resonant frequency of $29 \mathrm{GHz}$ in the "far to the I-slots' deepest field." The current paths develop to a resonant frequency as the evolutions through the slots of $32 \mathrm{GHz}$.Diagram 5. shows the results of the proposed system using a genetic algorithm.

\section{Conclusion}

Artificial intelligence is at its height. It seems to be well known that ML is not only theoretically safe but also strategically beneficial.The present state of artificial intelligence in communication networks is just scratching the surface, but there is no denying that this technology will be made effective in the future. From a theoretical standpoint, we have reviewed how the major ML principles apply to networking applications in this paper.Besides, illustrated an actual deep learning program that is presently being used in manufacturing. We expect a slew of impactful neural network models to develop later I'll be in the networking community. Only "read-only" network data connection is needed for descriptive and predictive applications.As a response, even at this experimental stage, they are safe and relatively easy to configure. Nevertheless, they have the potential to fix a wide range of problems using arbitrarily sophisticated data mining algorithms. The as the infrastructures for computer vision, big data, and ip based networking (SDN) evolve, the telecommunications and networking industry is ready to use them considering the problems imposed by ML applications. This will pave the way for "adaptive networks," that would change today's static network into a dynamic, programmable environment driven by analytics and intelligence.

\section{References}

[1] T. Janevski, "Traffic Analysis and Design of Wireless IP Networks", Artech House Inc., Boston, USA, 2003.

[2] ITU-T, Y.2001, "General overview of NGN", December 2004.

[3] ITU-T, Y-2002, "Overview of ubiquitous networking and its support in NGN", October 2009

[4] T. Mitchell, Machine Learning, McGraw Hill, 1997.

[5] Zikria, Y. B., Kim, S. W., Afzal, M. K., Wang, H., \& Rehmani, M. H. (2018). 5G Mobile services and scenarios: Challenges and solutions.

[6] Burden, F., \& Winkler, D. (2008). Bayesian regularization of neural networks. Artificial neural networks, 23-42.

[7] Kabalci, Y. (2019). 5G mobile communication systems: Fundamentals, challenges, and key technologies. In Smart Grids and Their Communication Systems (pp. 329-359). Springer, Singapore.

[8] Janevski, T. (2009, January). 5G mobile phone concept. In 2009 6th IEEE consumer communications and networking conference (pp. 1-2). IEEE. 
[9] Tudzarov, A., \& Janevski, T. (2011). Design for 5G mobile network architecture. International Journal of Communication Networks and Information Security, 3(2), 112-123.

[10] Ameerudden, M. R., \& Rughooputh, H. C. (2012). Hybrid BSCF Genetic Algorithms in the Optimization of a PIFA Antenna. International Journal of Machine Learning and Computing, 2(6), 746

[11] El Misilmani, H. M., \& Naous, T. (2019, July). Machine learning in antenna design: An overview on machine learning concept and algorithms. In 2019 International Conference on High-Performance Computing \& Simulation (HPCS) (pp. 600-607). IEEE. 